\title{
The Effect of electrodeposition parameters and morphology on the performance of Au nanostructures for the detection of As (III)
}

Ren, Baiyu; Jones, Lathe; Chen, Miao; Oppedisano, Daniel; Qiu, Dong; Ippolito, Samuel; Bhargava, Suresh https://researchrepository.rmit.edu.au/esploro/outputs/9921863408201341/filesAndLinks?institution=61RMIT_INST\&index=null

Ren, B., Jones, L., Chen, M., Oppedisano, D., Qiu, D., Ippolito, S., \& Bhargava, S. (2017). The Effect of electrodeposition parameters and morphology on the performance of Au nanostructures for the detection of As (III). Journal of the Electrochemical Society, 164(14), 1121-1128.

https://doi.org/10.1149/2.1261714jes

Document Version: Published Version

Published Version: https://doi.org/10.1149/2.1261714jes

Repository homepage: https://researchrepository.rmit.edu.au

(c) The Author(s) 2017. Published by ECS. This is an open access article distributed under the terms of the Creative Commons Attribution 4.0 License (CC BY, http://creativecommons.org/licenses/by/4.0/), which permits unrestricted reuse of the work in any medium, provided the original work is properly cited.

Downloaded On 2023/04/26 19:37:20 +1000 
Thank you for downloading this document from the RMIT Research Repository.

The RMIT Research Repository is an open access database showcasing the research outputs of RMIT University researchers.

RMIT Research Repository: http://researchbank.rmit.edu.aul

\section{Citation:}

Ren, B, Jones, L, Chen, M, Oppedisano, D, Qiu, D, Ippolito, S and Bhargava, S 2017, 'The Effect of electrodeposition parameters and morphology on the performance of Au nanostructures for the detection of As (III)', Journal of the Electrochemical Society, vol. 164, no. 14, pp. 1121-1128.

\section{See this record in the RMIT Research Repository at:}

https://researchbank.rmit.edu.au/view/rmit:49099

Version: Published Version

\section{Copyright Statement:}

(C) The Author(s) 2017. Published by ECS. This is an open access article distributed under the terms of the Creative Commons Attribution 4.0 License (CC BY, http://creativecommons.org/licenses/by/4.0/), which permits unrestricted reuse of the work in any medium, provided the original work is properly cited.

\section{Link to Published Version:}

https://dx.doi.org/10.1149/2.1261714jes 


\title{
The Effect of Electrodeposition Parameters and Morphology on the Performance of Au Nanostructures for the Detection of As (III)
}

\author{
Baiyu Ren, ${ }^{\mathrm{a}}$ Lathe A. Jones, ${ }^{\mathrm{a}, \mathrm{z}}$ Miao Chen, ${ }^{\mathrm{a}, \mathrm{b}}$ Daniel K. Oppedisano, ${ }^{\mathrm{a}, *}$ Dong Qiu, \\ Samuel J. Ippolito, ${ }^{\text {a,c }}$ and Suresh K. Bhargava ${ }^{a}$ \\ ${ }^{a}$ Centre for Advanced Materials and Industrial Chemistry (CAMIC), School of Science, RMIT University, \\ Melbourne, VIC 3001, Australia \\ ${ }^{b}$ CSIRO Mineral Resources, Clayton, VIC 3169, Australia \\ ${ }^{c}$ School of Engineering, RMIT University, Melbourne, VIC 3001, Australia
}

\begin{abstract}
A series of Au nanospikes and dendrites were electrodeposited with either an inorganic $\left(\mathrm{Pb}^{2+}\right)$ or organic (cysteine) growth directing agent for different times to obtain varied morphology. These structures were compared with gold nanoparticles of three different shapes (Octahedral, Cubic and Rhombic Dodecahedral) for detection of As (III) by Square Wave Anodic Stripping Voltammetry (SWASV). The sensitivity and limit of detection (LOD) was dependent on the surface crystallographic orientations and the morphology, with superior sensitivity confirmed with a maximum amount of Au (111) facets on the surface for the nanoparticles. XRD studies suggested that the shape directing influence of $\mathrm{Pb}^{2+}$ is lost on the gold nanospikes at higher deposition time, and that the size of the (111) terraces on the polycrystalline surfaces decreased, which led to a loss of performance. For gold dendrites, as the cysteine maintained shape directing behavior through hierarchical dendritic branching, deposition time did not affect the sensitivity. The study confirms that electrodeposition parameters or nanoparticle synthesis methods for Au surfaces in arsenic sensing needs to be carefully controlled, to either maximize the (111) facets, or minimize the steps on a polycrystalline Au surface, and that inorganic and organic shape directing agents will have differing effects.

(C) The Author(s) 2017. Published by ECS. This is an open access article distributed under the terms of the Creative Commons Attribution 4.0 License (CC BY, http://creativecommons.org/licenses/by/4.0/), which permits unrestricted reuse of the work in any medium, provided the original work is properly cited. [DOI: 10.1149/2.1261714jes] All rights reserved.

(cc) BY
\end{abstract}

Manuscript submitted October 6, 2017; revised manuscript received November 17, 2017. Published December 13, 2017.

Arsenic (As) is a naturally occurring toxic substance associated with adverse health effects, including mutagenicity and carcinogenity. ${ }^{1}$ Contamination of groundwater by arsenic has been reported in more than 20 countries $^{2}$ with a recommended $10 \mathrm{ppb}$ upper limit for drinking water set by the World Health Organization (WHO). ${ }^{3}$ Arsenite (As (III)) is considered the most toxic form in natural water, while Arsenate (As (V)) is 50 times less poisonous and generally the most stable in oxidising conditions. ${ }^{4-7}$ Anthropogenic emissions of arsenic from industrial effluents, combustion of fossil fuels, or mining of As containing ores are a significant source, as are natural origins such as As bearing minerals. ${ }^{8,9}$

A variety of conventional laboratory analytical techniques are available for arsenic detection including atomic absorption/fluorescence spectrometry, surface enhanced Raman scattering (SERS), high performance liquid/gas chromatography and inductively coupled plasma mass spectrometry (ICPMS). ${ }^{10}$ However, there remains a need for detection methods that are economically competitive, due to prevalent As in remote regions of the developing world, and such methods must be accurate and convenient for technicians to conduct on-site analysis. Electrochemical methods are useful to this end, ${ }^{11}$ and a large range of materials have been developed for electrochemical detection of arsenic. Gold-based materials have been studied extensively for electrochemical detection of Arsenic (As), with the technique of Anodic Stripping Voltammetry (ASV) offering excellent sensitivity and limit of detection. ${ }^{12}$ Nanostructured $\mathrm{Au}$ electrodes have been shown to have a lower limit of detection (LOD) and higher sensitivity than conventional gold electrodes due to their increased surface area.

It is well established that morphology and surface crystallographic orientation also has a significant effect on the electrochemical behavior of gold electrodes, especially for applications in catalysis. For example, oxidation of methanol on gold electrodes has been studied on $\mathrm{Au}$ (111), $\mathrm{Au}(100)$ and $\mathrm{Au}$ (110), concluding that the $\mathrm{Au}$ (110) orientation and $\mathrm{Au}$ (111) orientation favor electro-oxidation of methanol in alkaline media. ${ }^{13}$ Another example is the numerous studies on the oxygen reduction reaction (ORR) on gold single crystals and gold modified electrodes, showing $\mathrm{Au}(100)$ is more active than $\mathrm{Au}(110)$,

*Electrochemical Society Member

zE-mail: lathe.jones@rmit.edu.au and $\mathrm{Au}(111)$ is the least active in alkaline electrolyte. ${ }^{14-16}$ These are important electrocatalytic reactions that are intensely studied, but there has been less emphasis on the effect of morphology on stripping analysis, despite nanoparticles and nanostructures being commonly used to increase sensitivity. Systematic characterization of the nanomaterials used, and the effects of morphological variations are quite rare for such electroanalytical studies. For stripping methods, such as adsorptive stripping voltammetry of As (III), where As (III) is adsorbed and then reduced to As (0) on the electrode surface before anodic stripping, one may expect that the electrochemical behavior is intimately linked to the interaction of the As with the Au surface. This should vary for the different facets derived from crystal planes in the $\mathrm{Au}$ face centered cubic (fcc) crystal structure, or the morphology of the surface.

Single crystal surfaces usually reveal fundamental aspects of facetdependent behavior, but are not practical in real world sensors. Previously, it has been shown that an Au (111)-like electrode, fabricated from polycrystalline gold with a self-assembled monolayer (SAM) of n-butanethiol (n-BT) that selectively blocks the (110) and (100) $\mathrm{Au}$ facets, shows an improved selectivity for As (III) in the presence of $\mathrm{Cu}$ (II) compared to polycrystalline Au electrodes, ${ }^{17}$ indicating that surface crystallography of low index facets affects sensitivity. Recent studies on Au nanoparticles in $\mathrm{H}_{2} \mathrm{SO}_{4}$ solution gave further evidence that the $\mathrm{Au}$ (111) facet displays enhanced sensitivity, with different facet sensitivity ascribed to the deposition process during pre-concentration and the binding energy of the As on the surface of $\mathrm{Au} .{ }^{18} \mathrm{We}$ are thus interested in making physically and chemically stable electrochemical sensors with well-controlled surface morphology, and comparing the performance of such surfaces under the same conditions to model nanoparticles. A variety of chemical techniques have been developed to fabricate Au nanostructures with controlled shapes such as flower-like particles, ${ }^{19-22}$ textile-like sheet arrays, ${ }^{23}$ nanowires, ${ }^{24}$ nanobelts and nanocombs, ${ }^{25}$ nanoplates, ${ }^{26}$ polyhedral, ${ }^{27}$ hexagram shaped ${ }^{28}$ and dendrites. ${ }^{29-31}$ Electrodeposition is the most efficient technique for directly producing nanostructured surfaces onto conducting substrates that can be directly used as the sensor. Compared with AuNPs prepared by complex wet-chemical methods, which tend to aggregate due to high surface energy, and may show decreased activity due to the use of capping agents, directly electrodeposited gold structures may have cleaner surfaces, and more easily fabricated via a rapid and simple one-step electrodeposition procedure. Gold spikes 
have been developed by our group, exhibiting promising electrocatalytic and SERS properties. ${ }^{32,33}$ The controlled growth of spike-like Au structures using $\mathrm{Pb}^{2+}$ as a shape directing agent allows preferential growth in the $<111>$ direction due to the preference of $\mathrm{Pb}^{2+}$ for the (110) and (100) facets. Organic shape directing agents have also been used to electrodeposit dendritic structures, with hierarchal dendrites being formed as the electrodeposition progresses in time. ${ }^{34}$

In this paper, $\mathrm{Au}$ spikes with three different morphologies were obtained by undertaking electrodeposition for three different time periods. The electroanalytical performances of these three gold morphologies confirm that As (III) detection via ASV on Au is highly structure sensitive, and that conditions for electrodeposition need to be closely controlled when preparing surfaces for analysis. To confirm the relationship between Au surface structure and As (III) analysis, gold nanoparticles with controlled shapes, being octahedral $\left(\mathrm{AuNP} \mathrm{OCT}_{\mathrm{O}}\right)$, cubic $\left(\mathrm{AuNP}_{\mathrm{CUB}}\right)$, and rhombic dodecahedral $\left(\mathrm{AuNP}_{\mathrm{RD}}\right)$ bounded predominately by (111), (100) and (110) respectively, were synthesized and tested under the same conditions as the electrodeposited $\mathrm{Au}$ spikes. Finally, gold dendrites electrodeposited for three time periods in the presence of cysteine were tested in order to contrast the use of an inorganic or organic shape directing agent.

In this way we have increased our practical understanding of the factors that need to be controlled when designing Au nanostructured electrodes to maximize the sensitivity, and improve the LOD of As (III) electroanalysis in aqueous solution.

\section{Experimental}

Materials.- $\mathrm{Pb}\left(\mathrm{CH}_{3} \mathrm{COO}\right)_{2} \cdot 3 \mathrm{H}_{2} \mathrm{O}(99 \%)$, Gold (III) chloride trihydrate $\left(\mathrm{HAuCl}_{4} \cdot 3 \mathrm{H}_{2} \mathrm{O}, 99.9 \%\right)$, cetyltrimethylammonium bromide (CTAB, 99\%), L-ascorbic acid (99\%), sodium borohydride $\left(\mathrm{NaBH}_{4}\right.$, 99\%), cetyltrimethylammonium chloride (CTAC, $25 \mathrm{wt} \%$ in $\mathrm{H}_{2} \mathrm{O}$,), sodium bromide $(\mathrm{NaBr}, 99 \%)$, lead nitrate $(99 \%)$, nafion perfluorinated resin solution (5 wt\%), L-Cysteine and $\mathrm{As}_{2} \mathrm{O}_{3}(99 \%)$ were all obtained from Sigma Aldrich and used as received. All the solutions were prepared with Milli-Q (18 $\left.\mathrm{M} \Omega \mathrm{cm}^{-1}\right)$ deionized water. As (III) stock solution was prepared by dissolving $\mathrm{As}_{2} \mathrm{O}_{3}$ in concentrated $\mathrm{NaOH}$, and the $\mathrm{pH}$ was adjusted to 3.0 with concentrated $\mathrm{HNO}_{3}$ and then diluted with Milli-Q water. Solutions of other concentrations were prepared from this stock solution. Caution: Care should be taken when handling and disposing of As (III) solutions due to their toxicity.

Preparation of Au spikes, Au nanoparticles and Au dendrites.Gold spikes were electrodeposited on a $150 \mathrm{~nm}$ thick gold polycrystalline substrate (prepared by E-beam evaporation onto a Si base with a $10 \mathrm{~nm}$ Ti intermediate layer) from a solution containing $20.7 \mathrm{mM}$ $\mathrm{HAuCl}_{4}$ and $1 \mathrm{mM} \mathrm{Pb}\left(\mathrm{CH}_{3} \mathrm{COO}\right)_{2} .{ }^{35}$ The E-beam evaporated Au film was rinsed in acetone, ethanol and Milli-Q deionized water to remove possible contaminants, followed by drying in a stream of nitrogen gas prior to use. An inert graphite rod $(6 \mathrm{~mm}$ diameter, Johnson Matthey Ultra "F" purity grade) was used as the counter electrode with a $\mathrm{Ag} / \mathrm{AgCl}(3 \mathrm{M} \mathrm{NaCl}, 0.210 \mathrm{~V}$ vs SHE) reference electrode, and all potentials in this paper are referred directly to this reference electrode. Electrodeposition was undertaken at a constant potential of $0.05 \mathrm{~V}$ for 720 s, 1200s and 1800s, to generate the three Au spike morphologies. All solutions were degassed with a $\mathrm{N}_{2}$ stream for $10 \mathrm{~min}$ prior to each deposition, and a continuous $\mathrm{N}_{2}$ flow was maintained over the solution headspace during the electrodeposition process.

$\mathrm{Au}$ octahedral $\left(\mathrm{AuNP} \mathrm{OCT}_{\mathrm{OC}}\right)$ nanoparticles were prepared via a published method. ${ }^{36}$ In summary, a $20 \mathrm{~mL}$ solution of $0.125 \mathrm{mM} \mathrm{HAuCl}_{4}$ and $0.01 \mathrm{M}$ cetyltrimethylammonium bromide (CTAB) was prepared first. Thereafter a $100 \mu \mathrm{L}$ solution of $0.1 \mathrm{M} \mathrm{L}$-ascorbic acid was added to the $20 \mathrm{~mL}$ solution. Finally, $100 \mu \mathrm{L}$ solution of $0.1 \mathrm{M} \mathrm{NaOH}$ was quickly added. The color of the solution changed to purple-red within 20 minutes, indicating the formation of Au octahedral nanoparticles. The mixture was centrifuged at $7000 \mathrm{rpm}$ for $20 \mathrm{~min}$ and the solid was rinsed with Milli-Q water 3 times to remove the excess reactants.

$\mathrm{Au}$ cubic nanoparticles $\left(\mathrm{AuNP}_{\mathrm{CUB}}\right)$ were prepared by a seedmediated approach. ${ }^{37}$ A $10 \mathrm{~mL}$ aqueous seed solution was prepared by the reduction of $0.25 \mathrm{mM} \mathrm{HAuCl}_{4}$ by ice-cold $\mathrm{NaBH}_{4}(0.6 \mathrm{mM})$ in the presence of $0.075 \mathrm{M}$ cetyltrimethylammonium bromide (CTAB). The seed solution was used $2 \mathrm{~h}$ after its preparation. In the growth solution, $0.2 \mathrm{mM} \mathrm{HAuCl}_{4}$ was reduced by $0.009 \mathrm{M} \mathrm{L}$-ascorbic acid in the presence of $0.016 \mathrm{M} \mathrm{CTAB}$, followed by the addition of gold seed solution $\left(1.25 \times 10^{-8} \mathrm{M}\right)$. The solution was gently shaken after the addition of every component and was left to sit for 8 hours. Thereafter the solution containing cubic Au nanoparticles was centrifuged at $5000 \mathrm{rpm}$ for $10 \mathrm{~min}$ and rinsed with Milli-Q water 3 times.

Rhombic dodecahedra Au nanoparticles $\left(\mathrm{AuNP}_{\mathrm{RD}}\right)$ were prepared following a two-step procedure. ${ }^{38}$ For gold seeds, a $10 \mathrm{~mL}$ solution of $0.25 \mathrm{mM} \mathrm{HAuCl}_{4}$ and $0.1 \mathrm{M}$ cetyltrimethylammonium chloride (CTAC) was prepared. To this solution, ice-cold $\mathrm{NaBH}_{4}$ solution $(0.45$ $\mathrm{mL}, 0.02 \mathrm{M}$ ) was added with stirring. The resulting brown solution was left for $2 \mathrm{~h}$ before use. Two vials labelled $\mathrm{A}$ and $\mathrm{B}$ were used for the growth solution. In each vial $250 \mu \mathrm{L}$ of $0.01 \mathrm{M} \mathrm{HAuCl}_{4}$ solution, $0.3306 \mathrm{~mL}$ of CTAC, $10 \mu \mathrm{L}$ of $0.01 \mathrm{M} \mathrm{NaBr}$ and $9.2344 \mathrm{~mL}$ of Milli$\mathrm{Q}$ water were mixed first. To both vials $\mathrm{L}$-ascorbic acid $(150 \mu \mathrm{L}, 0.04$ $\mathrm{M})$ was added then and the solution became colorless. Afterwards, 25 $\mu \mathrm{L}$ of the gold seeds was added to vial A with shaking. The mixture in vial A turned light pink in 5 seconds. Then $25 \mu \mathrm{L}$ of the solution in vial A was added to vial B. The solution in vial B was left to sit for $20 \mathrm{~min}$ for the completion of the particle growth, and subsequently centrifuged at $4000 \mathrm{rpm}$ for $10 \mathrm{~min}$ and rinsed twice before collection of the nanoparticles.

$\mathrm{Au}$ dendrites were electrodeposited on glassy carbon (GC) electrode following a published procedure. ${ }^{34}$ Briefly, a GC electrode was firstly polished on a polishing cloth with $0.05 \mu \mathrm{m}$ alumina slurry, followed by ultrasonication in ethanol and water for $10 \mathrm{~min}$ in sequence. $\mathrm{Au}$ dendrites were electrodeposited at $-0.8 \mathrm{~V}$ for $1000 \mathrm{~s}, 2000 \mathrm{~s}$ and $3000 \mathrm{~s}$ respectively in the presence of $1 \mathrm{mM} \mathrm{HAuCl}_{4}+0.1 \mathrm{mM}$ cysteine in $0.5 \mathrm{M} \mathrm{H}_{2} \mathrm{SO}_{4}$. The as-prepared $\mathrm{Au}$ dendrites were pre-treated at $-1.3 \mathrm{~V}$ for $120 \mathrm{~s}$ in $0.5 \mathrm{M} \mathrm{HNO}_{3}$ to remove cysteine adsorbed on all facets before being used for analysis of As (III).

Electrochemical characterization.-Au nanoparticles were studied on modified glassy carbon (GC) electrodes, with a GC geometric area of $0.2376 \mathrm{~cm}^{2}$, which was polished with $0.05 \mu \mathrm{m}$ alumina slurry followed by sonication in ethanol and Milli-Q water for $10 \mathrm{~min}$, respectively. The GC electrodes were then treated with a $10 \mu \mathrm{L}$ aliquot of the $\mathrm{Au}$ nanoparticle solution that was directly dropped onto the surface. Au nanoparticle/GC electrodes were left to dry naturally and then covered by $10 \mu \mathrm{L}$ of $0.5 \mathrm{wt} \%$ nafion solution to increase the stability of $\mathrm{Au}$ nanoparticles on the surface.

Electrochemical analysis was undertaken using a $\mathrm{CH}$ Instruments (CHI 760C) electrochemical analyzer with a standard three-electrode system. Working electrodes were either the gold spike electrodes or $\mathrm{Au}$ dendrite electrodes (used directly) or $\mathrm{Au}$ nanoparticle (AuNP) modified GC electrodes. A platinum wire and an $\mathrm{Ag} / \mathrm{AgCl}$ (3 M $\mathrm{NaCl}$ ) electrode were used as the auxiliary electrode and reference electrode, respectively.

Cyclic voltammetry (CV) was conducted for lead underpotential deposition (UPD) to probe the exposed low index surface facets of the Au working electrodes from $-0.2 \mathrm{~V}$ to $-0.7 \mathrm{~V}$ in $1 \mathrm{mM} \mathrm{Pb}(\mathrm{NO})_{3}+0.1$ $\mathrm{M} \mathrm{NaOH}$ at $50 \mathrm{mV} \mathrm{s}^{-1}$. Prior to lead UPD characterization, cyclic voltammetry was conducted from $-0.75 \mathrm{~V}$ to $0.9 \mathrm{~V}$ at $50 \mathrm{mV} \mathrm{s}^{-1}$ to clean the electrode by $\mathrm{PbO}_{2}$ deposition and stripping. ${ }^{39,40}$

Square Wave Adsorptive Stripping Voltammetry (SWASV) was utilized for As (III) detection. For Au spike or dendrite electrodes, As $(0)$ was pre-deposited at a potential of $-0.35 \mathrm{~V}$ for $300 \mathrm{~s}$ or 100 $\mathrm{s}$, followed by As stripping between $-0.35 \mathrm{~V}$ and $0.7 \mathrm{~V}$, with the following parameters: frequency, 50 or $15 \mathrm{~Hz}$; amplitude, $0.025 \mathrm{~V}$; potential increment, $0.004 \mathrm{~V}$. For AuNP/GC electrodes, the measurement involved: (i) Pre-deposition of arsenic at a potential of $-0.35 \mathrm{~V}$ for either $60 \mathrm{~s}, 100 \mathrm{~s}$, or $150 \mathrm{~s}$. (ii) A SWASV scan (anodic stripping) from $-0.35 \mathrm{~V}$ to $0.7 \mathrm{~V}$ with an increment of $0.004 \mathrm{~V}$, an amplitude of $0.025 \mathrm{~V}$ and a frequency of $40 \mathrm{~Hz}$. The sensitivity $\left(\mathrm{uA} \mathrm{cm}{ }^{-2} \mathrm{ppb}^{-1}\right)$ was determined by the slope of the calibration curve for As stripping, corrected for the electrochemical surface area (ECSA) of the Au 

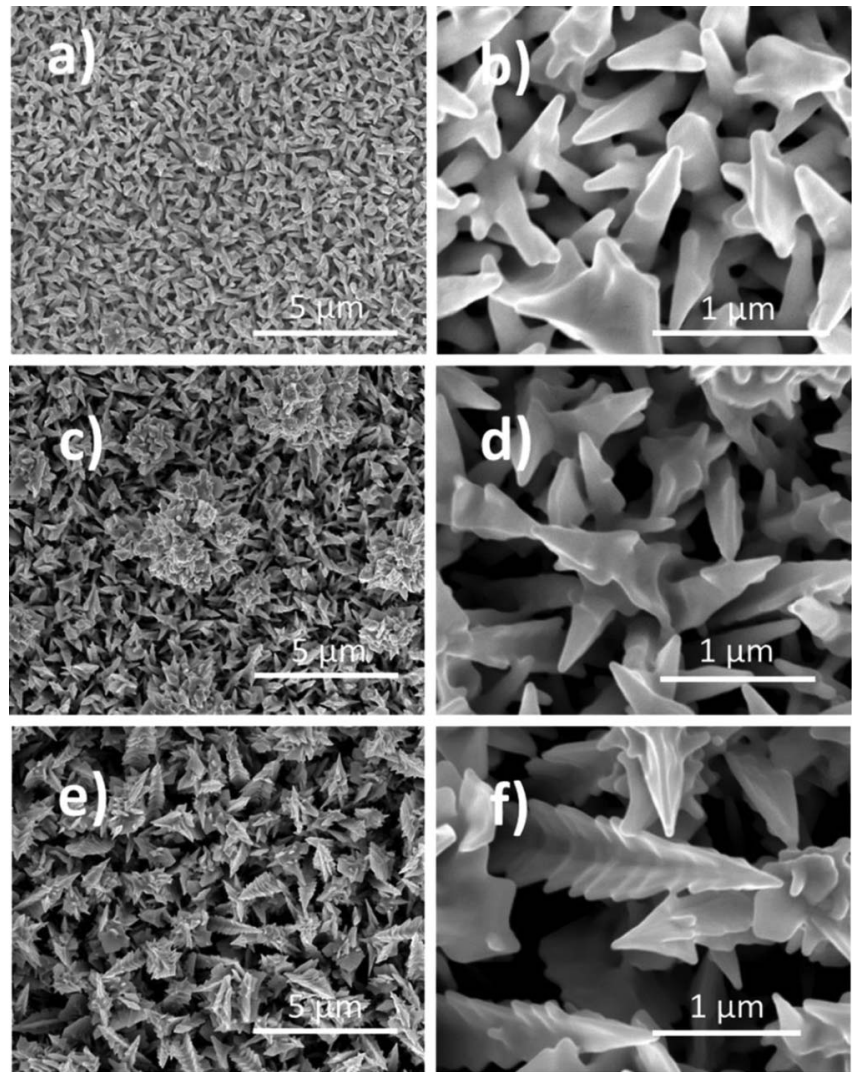

Figure 1. SEM images of gold spikes electrodeposited on gold substrates at $0.05 \mathrm{~V}$ for various time: $720 \mathrm{~s}(\mathrm{a}, \mathrm{b}), 1200 \mathrm{~s}(\mathrm{c}, \mathrm{d}), 1800 \mathrm{~s}(\mathrm{e}, \mathrm{f})$.

surface, which was determined by voltametric analysis in acid, assuming a charge density of $400 \mu \mathrm{C} \mathrm{cm}^{-2} \cdot{ }^{41-43}$ The limit of detection (LOD) was estimated by standard deviation multiplied by 3 divided, by the sensitivity, utilising the standard deviation of the blank solution under identical conditions for analysis. ${ }^{44,45}$

Instrumentation.-Scanning electron microscopy (SEM) was performed on a FEI Verios 460L SEM with a working distance of $4 \mathrm{~mm}$ and a voltage of $1 \mathrm{kV}$. Electron backscattered diffraction (EBSD) patterns were recorded under an accelerating voltage of $15 \mathrm{KV}$ on an FEI Nova SEM instrument using an Oxford NordlysMax ${ }^{2}$ EBSD Detector with Oxford Instruments Aztec software. The orientation of each individual grain and the overall inverse pole figures were obtained using an HKL Channel 5 EBSD system. Prior to SEM imaging and EBSD analysis, samples were thoroughly rinsed with Milli-Q water and dried under a flow of nitrogen. Transmission electron microscopy (TEM) and selected-area electron diffraction (SAED) measurements were performed on a JEOL 1010 TEM at $100 \mathrm{kV}$ accelerating voltage. Grazing incidence small angle X-ray diffraction (GID) patterns were obtained with a Bruker AXS D8 Discover with General Area Detector Diffraction System (GADDS) using $\mathrm{Cu} K \alpha$ radiation $(\lambda=1.5406 \AA)$ and a $\omega$ of 1 degree.

\section{Results and Discussion}

Growth directing agents such as $\mathrm{Pb}^{2+}$ tend to lead to a predominate crystallographic growth direction during electrodeposition. In this study we varied deposition time to create a series of structures for As (III) sensing. Typical SEM images of the Au spikes electrodeposited for three different times are shown in Figures 1a-1f, where visual variations in morphology are observed for these three deposition times. The three Au spike morphologies all have an extremely uniform coverage, with outward growth of spike-shaped gold from the substrate. The spikes deposited for $720 \mathrm{~s}$ (Figures 1a, 1b) are up to $500 \mathrm{~nm}$ long with $50 \mathrm{~nm}$ diameter bases and well-defined tips that taper to approximately $10 \mathrm{~nm}$ diameter. When the deposition time increased to $1200 \mathrm{~s}$, the lengths of spikes are increased to $\sim 1 \mu \mathrm{m}$, with a thicker core structure and some "barbed" morphology, as shown in Figures $1 \mathrm{c}$ and $1 \mathrm{~d}$. Figures $1 \mathrm{e}$ and 1f show thicker gold spikes with longer tips of $2-3 \mu \mathrm{m}$, as well as the appearance of more barbs along the backbone of gold spikes when the deposition time is increased to $1800 \mathrm{~s}$. We do not see hierarchical dendritic branching as observed in growth directed by organic agents for $\mathrm{Au}$ dendrites under similar conditions, ${ }^{34}$ but rather a thicker spike with a barbed structure as more $\mathrm{Au}$ is deposited on the surface. Cyclic voltammetric curves were first recorded in $0.5 \mathrm{M} \mathrm{H}_{2} \mathrm{SO}_{4}$ to probe the surface of the electrodeposited $\mathrm{Au}$ spikes. The corresponding profiles are shown in Figure S1 (see Supporting Information). Voltammograms for all gold spike electrodes show features typical of $\mathrm{Au}$, with oxidation features at 1-1.5 $\mathrm{V}$ and corresponding reduction peaks at $\sim 0.9 \mathrm{~V} .^{22,46}$ Electrochemical Surface Area (ECSA) based on the reduction peaks were calculated to be $0.136,0.648,0.922$ and $1.63 \mathrm{~cm}^{2}$ for Au substrate, $720 \mathrm{~s}, 1200$ $\mathrm{s}$ and $1800 \mathrm{~s}$ Au spikes respectively, confirming an increase of ECSA in the gold spikes with the increase of deposition time as expected.

Analysis of As (III) on the Au spikes was carried out via Square Wave Anodic Stripping Voltammetry (SWASV) in $0.5 \mathrm{M} \mathrm{HNO}_{3}$. SWASV responses of the three gold spike electrodes with different concentrations of As (III) are shown in Figures 2a-2c. The corresponding calibration plots from $7-40 \mathrm{ppb}$ for As (III) are plotted in Figure 2d, indicating good linearity for all three gold spikes in this concentration range. The calibration plots clearly show, when corrected for surface area, that gold spikes with a deposition time of 720 s display the best sensitivity, with the highest slope, while the 1800 s gold spikes obtained the lowest sensitivity of the three gold spike morphologies. A further increase of As (III) concentration leads to an increase in peak currents for all three gold spikes and the corresponding calibration curves in the broader concentration range are shown in Figure S2 (see Supporting Information). It is observed that all gold spikes eventually show a leveled-off calibration curve with the increase of As (III) concentrations, due to the saturation of the gold surface. The linearity range increases from $720 \mathrm{~s}$ gold spikes to $1800 \mathrm{~s}$ gold spikes, due to the increase of surface area, however this could be circumvented by using a shorter pre-concentration time for the $720 \mathrm{~s}$ spikes, as the sensitivity is clearly superior. The LOD of the three gold spikes were $0.42,2.64$, and $5.64 \mathrm{ppb}$ for $720 \mathrm{~s}, 1200$ s and 1800 s gold spikes, respectively. The sensitivity and limit of detection (LOD) of the gold spike electrodes for As (III) determination are stated in Table I. It indicates that the Au spikes deposited for $720 \mathrm{~s}$ have the highest sensitivity of $0.15 \mu \mathrm{A} \mathrm{cm} \mathrm{cmb}^{-1}$ and the lowest Limit of detection (LOD) of $0.42 \mathrm{ppb}$ under these conditions.

Detailed characterization of the gold spikes was performed to understand the differences in their structures and surface crystallography. Due to the presence of a gold film beneath the gold spikes, conventional X-Ray diffraction (XRD) showed interference from the substrate. Grazing incidence small angle X-ray diffraction (GID) is much more surface sensitive and was thus utilized in this case. Unsurprisingly, five XRD diffraction peaks, indexed to the (111), (200), (220), (311), and (222) reflections of face-centered cubic (fcc) gold were observed for each of the gold spike morphologies, confirming a high quality $\mathrm{Au}$ crystalline structure. The GID patterns recorded in Figure S3a (see Supporting Information) confirm that the interference of gold substrate can be ignored due to the low intensities of $\mathrm{Au}$ (111) and $\mathrm{Au}(200)$ in the blank. The ratio of the intensities of the (111) reflections relative to $(200)\left(I_{111} / I_{200}\right)$ was 1.30 for the $720 \mathrm{~s}$ spikes, indicating crystallites in this structure are significantly elongated in the $<111>$ direction due to directed growth induced by the $\mathrm{Pb}^{2+}$ in solution, which is less strongly adsorbed on to the (111) facet. As the deposition time increases, the ratio $\left(\mathrm{I}_{(111)}\right) /\left(\mathrm{I}_{(200)}\right)$ decreases to 1.22 $(1200 \mathrm{~s})$ and $1.16(1800 \mathrm{~s})$. The ratios of the diffraction peaks are presented in Table $\mathrm{S} 1$. This result suggests that as the growth proceeds and barbed morphologies appear on the surface of the thickening spike, $\mathrm{Pb}^{2+}$ in solution loses its effectiveness as a growth directing agent, 

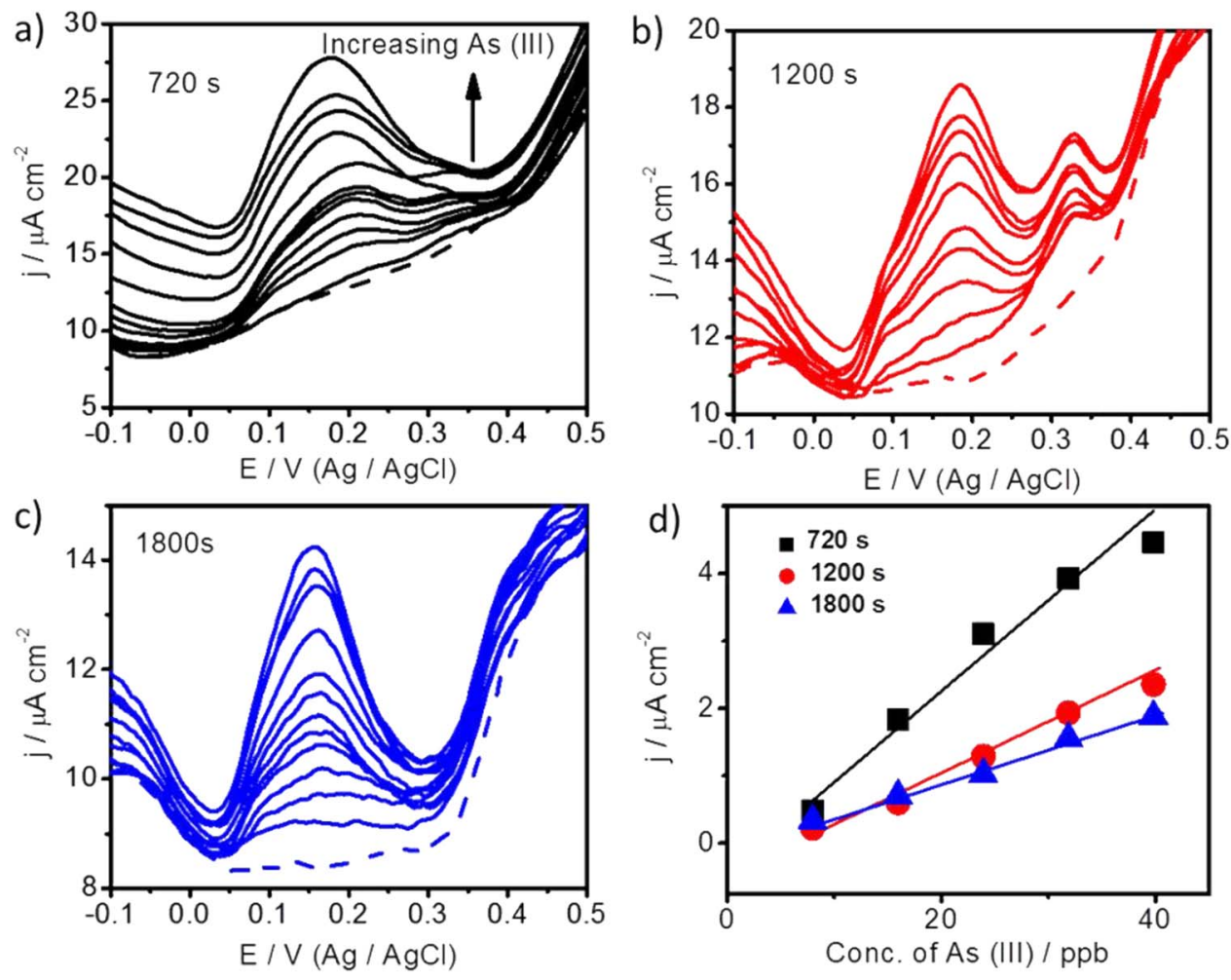

Figure 2. SWASV responses of the gold spikes electrodes deposited for: a) $720 \mathrm{~s}, \mathrm{~b}) 1200 \mathrm{~s}$, c) $1800 \mathrm{~s}$ for As (III) analysis in $0.5 \mathrm{M} \mathrm{HNO}_{3}$. The dash line refers to the baseline. d) Calibration curves of current density vs. concentrations of As (III) for the three Au spike electrodes. Deposition potential, $-0.35 \mathrm{~V}$; deposition time, $300 \mathrm{~s}$; initial potential, $-0.35 \mathrm{~V}$; final potential, $0.7 \mathrm{~V}$; frequency, $50 \mathrm{~Hz}$; amplitude, $0.025 \mathrm{~V}$; potential increment, $0.004 \mathrm{~V}$.

and the increase in thickness of the spikes is not a result of anisotropic growth. This allows us to generate stable structures that vary in their proportion of bulk (111) in the spikes. This decrease of (111) intensity in diffraction patterns from 720 s to the longer deposition times leads to the 1800 s spikes being quite similar to electrodeposition of a polycrystalline material without a growth directing agent.

To further understand the crystalline structures of the gold spikes, EBSD was undertaken on a polished cross section of Au spikes. Inverse pole diagrams in Figure S3b (see Supporting Information) show that orientations in $720 \mathrm{~s}$ gold spikes mostly concentrate on the (111) corner while increasing deposition time to $1200 \mathrm{~s}$ and $1800 \mathrm{~s}$ (Figure S3c,d in Supporting Information) leads to dispersions to all facets. It further confirms that gold spikes progress from a predominately (111) orientation at $720 \mathrm{~s}$ to a mixed orientation at 1200 and $1800 \mathrm{~s}$. Similar results have been seen for electrodeposition of nanoparticles, with shorter electrodeposition times leading to materials rich in $\mathrm{Au}$ (111) domains, while longer electrodeposition times result in polycrystalline orientations. ${ }^{47}$ In this case as growth proceeds and surface area increases, the shape directing effect of the $\mathrm{Pb}^{2+}$ is less effective, and polycrystalline growth is responsible for the thickening of the spikes.
As the stripping behavior of As (III) on gold is a surface based phenomenon, it is necessary to directly confirm which surface facets are exposed to solution. Pb UPD is an effective technique to probe the surface crystallographic structures of gold materials and has been extensively studied on polycrystalline and single crystalline gold materials. ${ }^{48-50}$ Since the lead UPD process is stucture sensitive, the potentials of the lead deposition and stripping peaks are used to assist with identification of the relative energy of facets on the surface of the gold spikes in contact with solution. ${ }^{40,51}$ Figure S4 (see Supporting Information) depicts the cyclic voltammograms of the gold spikes in an alkaline solution of lead (II) nitrate with a broad potential window. A broad anodic peak from $0.1 \mathrm{~V}$ due to the deposition of a monolayer of $\mathrm{PbO}_{2}$ was observed, followed by another anodic feature between $0.6 \mathrm{~V}$ and $0.9 \mathrm{~V}$, which arises from the formation of a multilayer of $\mathrm{PbO}_{2}{ }^{39,52}$ The $\mathrm{PbO}_{2}$ film is stripped away on the reverse scan, showing a cathodic peak at $0.1 \mathrm{~V}$. The anodic and cathodic features in the range of $-0.2 \mathrm{~V}$ to $-0.7 \mathrm{~V}$ correspond to the lead UPD region. Figure 3 reveals the anodic stripping curves of the lead UPD region for the three gold spike morphologies. The gold spikes in this paper contain not only lower index facets but higher index facets along with terraces, steps and kinks, and are far more complicated than ideal single

Table I. Electrochemical sensitivity and Limit of detection for As (III) detection by SWASV.

\begin{tabular}{llll} 
& Surface Au domains & Sensitivity $\mu \mathrm{A} \mathrm{cm}^{-2} \mathrm{ppb}^{-1}$ & LOD ppb \\
\hline AuNP OCT & $(111)>(110)$ & $0.53 \pm 0.020$ & $0.28 \pm 0.015$ \\
AuNP RD & $(110)>>(111)$ & $0.25 \pm 0.023$ & $1.33 \pm 0.122$ \\
AuNP CUB & $(100)>(110),(111)$ & $0.09 \pm 0.004$ & $4.14 \pm 0.197$ \\
Au Spikes 720 s & Polycrystalline & $0.15 \pm 0.007$ & $0.42 \pm 0.020$ \\
Au Spikes 1200 s & Polycrystalline & $0.07 \pm 0.006$ & $2.64 \pm 0.232$ \\
Au Spikes 1800 s & Polycrystalline & $0.04 \pm 0.004$ & $5.64 \pm 0.633$ \\
Au dendrites 1000 s & Polycrystalline & $0.10 \pm 0.004$ & $5.87 \pm 0.184$ \\
Au dendrites 2000 s & Polycrystalline & $0.10 \pm 0.004$ & $5.38 \pm 0.693$ \\
Au dendrites $3000 \mathrm{~s}$ & Polycrystalline & $0.10 \pm 0.017$ & $5.54 \pm 0.764$
\end{tabular}




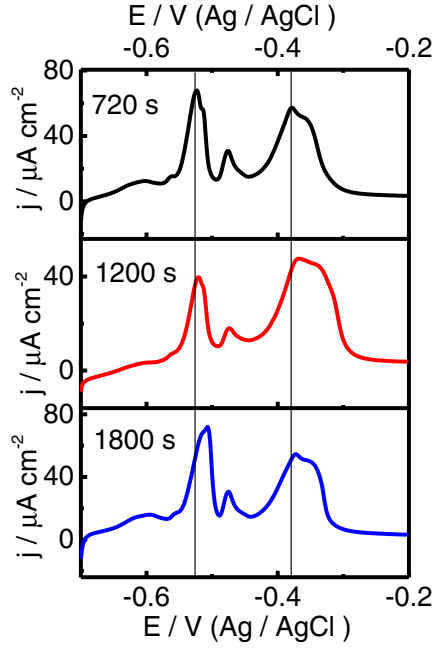

Figure 3. Voltammetric profiles of the gold spikes in $0.1 \mathrm{M} \mathrm{NaOH}+10^{-3} \mathrm{M}$ $\mathrm{Pb}\left(\mathrm{NO}_{3}\right)_{2}$ in the UPD stripping region. Scan rate: $50 \mathrm{mV} \mathrm{s}^{-1}$.

crystalline gold structures. Simply ascribing the three main anodic features at about $-0.523 \mathrm{~V},-0.476 \mathrm{~V}$ and $-0.378 \mathrm{~V}$ to $\mathrm{Au}(111)$, $\mathrm{Au}(100)$ and $\mathrm{Au}(110)$ crystal surfaces, respectively, ${ }^{39}$ is thus not possible. The $\mathrm{Pb}$ stripping does confirm that the surface is polycrystalline, as exposure of one facet would only be possible with a single crystal or high quality nanoparticle. In fact, all anodic peaks can be deconvoluted into smaller peaks. For example, the anodic stripping feature at about $-0.523 \mathrm{~V}$ for the $720 \mathrm{~s}$ spikes shows similar splitting peaks to literature reports in the (111) region. ${ }^{53,54}$ It is assumed that these peaks are the combinations of several smaller peaks resulting from some other higher index facets on the polycrystalline surface, ${ }^{55}$ though the peak height at $-0.523 \mathrm{~V}$ can considered as a resonable estimation for $\mathrm{Au}(111)$ and the highest current density value at -0.523 $\mathrm{V}$ ascertains the highest ratio of $\mathrm{Au}$ (111) facets in $720 \mathrm{~s}$ spikes. Furthermore, the most positive anodic peaks at about -0.378 is due to the overlapping of several peaks, which result from the large variety both/either the monoatomic steps of (111) faces and/or terraces, steps and kinks of other faces. ${ }^{55}$ An interesting feature is the anodic shift in the peak at approximately $-0.52 \mathrm{~V}$ at higher deposition time. The positive shift of this anodic feature for $1200 \mathrm{~s}$ and $1800 \mathrm{~s}$ spikes is marked as a vertical line in Figure 3. This may be interpreted as the generation of smaller $\mathrm{Au}(111)$ terraces as the spikes are deposited at longer times and the growth directing ability of the $\mathrm{Pb}^{2+}$ is lost. The superior sensitivity, as observed visually on the calibration curve for the 720 s spikes with a higher slope, and lower LoD than the spikes electrodeposited for longer times, are thus possibly due the 720s surface having larger $\mathrm{Au}$ (111) terraces. This is revealed by the lower energy stripping of $\mathrm{Pb}^{2+}$ in the upd region, with fewer kinks and steps generated on the surface at the shorter deposition time.

To further investigate the relationship between $\mathrm{Au}$ facets and As (III) sensing under the same conditions as the Au spikes, three different shapes of nanoparticles were synthesized: octahedral $\left(\mathrm{AuNP}_{\mathrm{OCT}}\right)$, cubic $\left(\mathrm{AuNP} \mathrm{CUB}_{\mathrm{B}}\right)$ and rhombic dodecahedral $\left(\mathrm{AuNP}_{\mathrm{RD}}\right)$ nanoparticles bounded predominately by $\mathrm{Au}(111), \mathrm{Au}(100)$ and $\mathrm{Au}$ (110) facets respectively, which allow a relatively precise surface facet control during SWASV analysis of As (III). Figures 4a and 4b show SEM and TEM images of orderly distributed AuNP $_{\text {OCT }}$, with an edge length of $\sim 27$ $\mathrm{nm}$. Larger scale SEM images in Figure S5a (see Supporting Information) indicates these gold nanoparticles display good monodispersion. The SAED pattern in Figure S5b (see Supporting Information) shows the AuNP ${ }_{\text {OCT }}$ are single crystals bounded by (111) facets. SEM and TEM images of cubic gold nanoparticles are shown in Figures 4d, 4e, which indicate a high-yield synthesis of monodispersed cubic gold nanoparticles of an edge length of $\sim 77 \mathrm{~nm}$, with some truncation on the edges and sides. The corresponding SAED pattern of a single cu- bic gold nanoparticle is shown in Figure s5d, suggesting the AuNP ${ }_{C U B}$ is a single crystal bounded by (100) facets. Figures $4 \mathrm{~g}$, $4 \mathrm{~h}$ show the SEM and TEM images of randomly distributed RD nanoparticles with an edge length of $\sim 75 \mathrm{~nm}$. Corresponding SAED pattern (Figure S5f in Supporting Information) further confirms the RD shapes bounded by 12 equivalent rhombic faces.

The cyclic voltammograms of modified GC electrodes of the gold nanoparticles are shown in Figure S6 (see Supporting Information) in $0.5 \mathrm{M} \mathrm{H}_{2} \mathrm{SO}_{4}$, and were used to determine the electrochemically active surface area (ECSA) for the modified electrodes. To estimate the effect of structure of the gold nanoparticles on the sensitivity for As (III) detection, calibration curves at a range of As (III) concentrations were taken. Figures $5 \mathrm{a}-5 \mathrm{c}$ show the SWASV curves for $\mathrm{AuNP}_{\mathrm{OCT}}, \mathrm{AuNP}_{\mathrm{RD}}$ and $\mathrm{AuNP} \mathrm{CUB}_{\mathrm{C}}$ modified GC electrodes after preconcentration of As $(0)$ for $100 \mathrm{~s}$ at $-0.35 \mathrm{~V}$. On the anodic scans, intense peaks (stripping currents) corresponding to the reoxidation of As (0) to the parent As (III) were observed for all nanoparticle shapes. Figure 5d shows the corresponding calibration plots for the AuNP modified GC electrodes within the upper end of their linear range. All AuNPs calibration curves show 2 different regimes, a linear region, and a levelled-off region at higher concentrations of As (III) due to saturation effects. This saturation occurs when the gold surface starts to become almost completely covered by the As( 0$)$ deposited during the pre-concentration step. The results indicate that the $\mathrm{AuNP}_{\mathrm{OCT}}$ modified electrode obtains the highest sensitivity of $0.53 \mu \mathrm{A} \mathrm{cm}$ $\mathrm{ppb}^{-1}$, while AuNP $\mathrm{CUB}_{\mathrm{B}}$ has the lowest sensitivity of $0.09 \mu \mathrm{A} \mathrm{cm}^{-2}$ $\mathrm{ppb}^{-1}$ and $\mathrm{AuNP}_{\mathrm{RD}}$ show a sensitivity of $0.25 \mu \mathrm{A} \mathrm{cm} \mathrm{cm}^{-2} \mathrm{ppb}^{-1}$. The LODs $(\mathrm{S} / \mathrm{N}=3)$ for octahedral, $\mathrm{RD}$ and cubic gold nanoparticles are calculated to be $0.28 \mathrm{ppb}, 1.33 \mathrm{ppb}$ and $4.14 \mathrm{ppb}$, respectively. In this case, the $\mathrm{AuNP}_{\mathrm{OCT}}$ modified electrode, when treated to the same deposition and stripping procedure at zero or very low As (III) concentration, has a cleaner background, with better discrimination of the stripping current, and responds with a higher slope to additions of As (III) on the calibration curve for the stripping current. We can conclude the performance for As (III) by SWASV in $\mathrm{HNO}_{3}$ of nanogold materials in this order: $\mathrm{AuNP}_{\mathrm{OCT}}>\mathrm{AuNP}_{\mathrm{RD}}>\mathrm{AuNP}_{\mathrm{CUB}}$, confirming $\mathrm{Au}$ (111) facet is more favorable than $\mathrm{Au}$ (110) and $\mathrm{Au}$ (100) for As (III) detection. Table I indicates the predominate surface facets, but also the secondary facets expected due to the truncated edges of the nanoparticles that were synthesized in practice, conceding that the nanoparticle shapes are not perfect.

Additional preconditioning times for As (III) reduction at -0.35 $\mathrm{V}$ were also undertaken. For example, As (III) was deposited at -0.35 $\mathrm{V}$ for $60 \mathrm{~s}$ (shorter than the $100 \mathrm{~s}$ ) and also $150 \mathrm{~s}$ (longer time), and the results are shown in Figure S7 and S8, respectively (see Supporting Information). The calibration plots for the shorter preconditioning time clearly indicates the $\mathrm{AuNP}_{\text {OCT }}$ modified GC electrode still has the highest sensitivity toward arsenic detection while AuNP $_{\text {CUB }}$ has the lowest sensitivity. For a higher As pre-concentration time of 150 $s$ there is an enhancement in current density of the stripping peak at the same concentration of As (III) compared with the shorter predeposition time, due to the higher amount of As (0) deposited onto the gold surface. In addition, the same trend is observed for the relative sensitivity of the three AuNP modified electrodes. A calibration curve for the AuNP electrodes at lower concentration (up to $130 \mathrm{ppb}$ ) is included in figure S12 (supporting information), showing linearity at lower concentration, and allowing direct comparison with the Au nanospike calibration curves.

For comparison with the Au spikes and nanoparticles, gold dendrites were prepared with the organic directing agent cysteine, since it has been reported that the presence of cysteine induces anisotropic growth during electrodeposition. ${ }^{34}$ It is well-known that the $\mathrm{SH}$ group of cysteine can adsorb on Au surfaces through strong Au-S bonds, which forms an insulating layer inhibiting the deposition and growth of Au. Figure S9 in the Supporting Information shows the adsorbed thiols can be desorbed through electrochemical reactions at a potential which depends on the crystallographic domains of the Au surface. Thus, the applied potential at $-0.8 \mathrm{~V}$ used for electrodeposition enables cysteine removed from $\mathrm{Au}$ (111) domains selectively over $\mathrm{Au}$ 

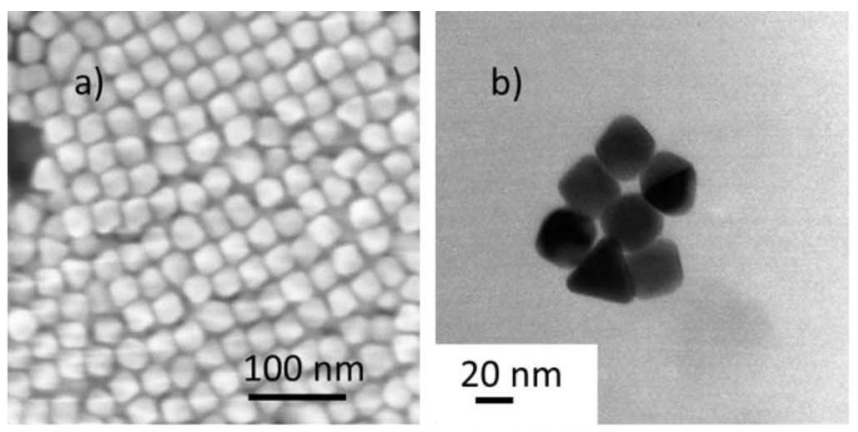

c)

\section{$\Delta \nabla$}

f)

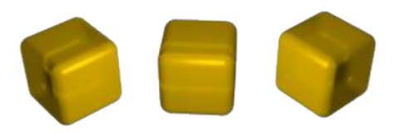

Figure 4. (a, d, g) SEM, (b, e, h) TEM images and (c, f, i) geometrical models of (a-c) octahedral, (d-f) cubic and (g-i) RD AuNPs at different magnifications.

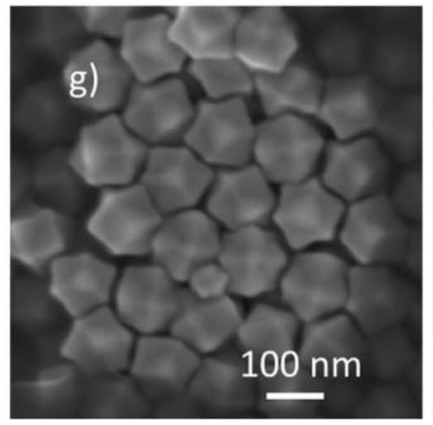

e)
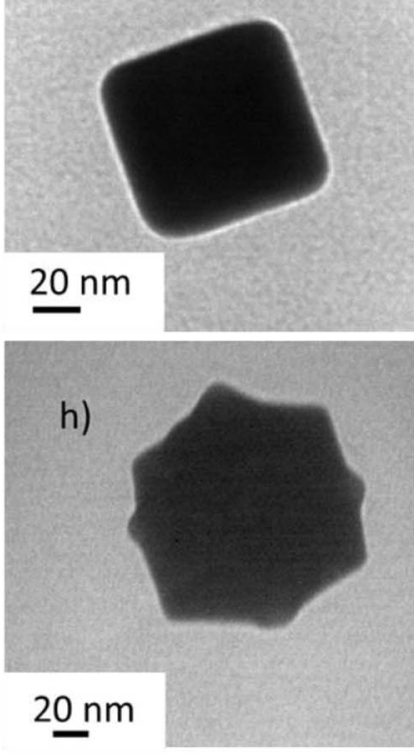

i)

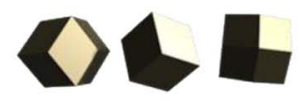

a)
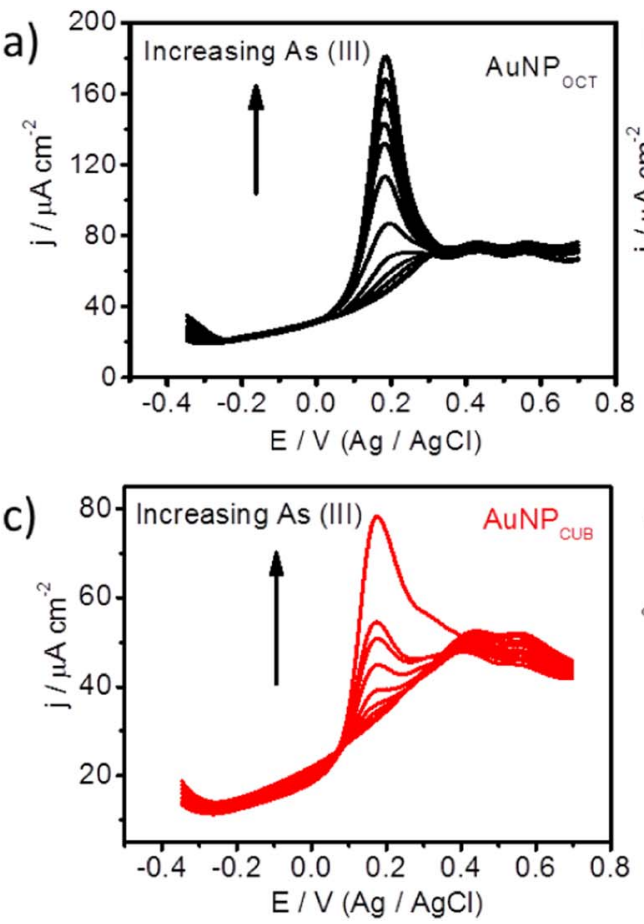
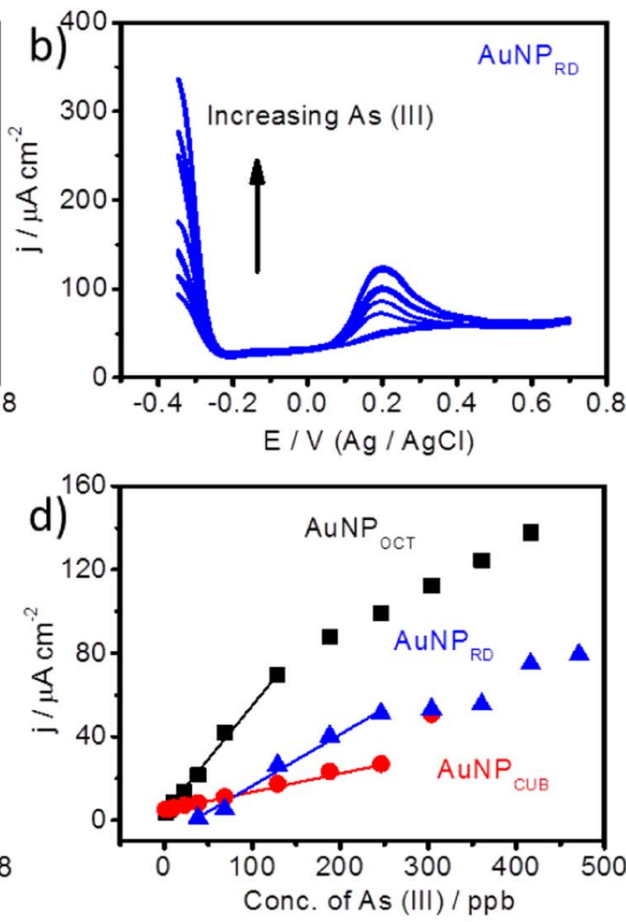

Figure 5. a-c) SWASV responses of the $\mathrm{AuNP}_{\mathrm{OCT}}, \mathrm{AuNP}_{\mathrm{RD}}$, and AuNP $\mathrm{CUB}_{\mathrm{B}}$ modified GC electrode toward As (III) at different concentrations in $0.5 \mathrm{M} \mathrm{HNO}_{3}$. d) Calibration curves of current density vs. concentration of As (III) for octahedral (dark), RD (blue) and cubic (red) gold nanoparticles modified GC electrodes. Deposition potential, -0.35 $\mathrm{V}$; deposition time, $100 \mathrm{~s}$; initial potential, $-0.35 \mathrm{~V}$; final potential, $0.7 \mathrm{~V}$; frequency, 40 $\mathrm{Hz}$; amplitude, $0.025 \mathrm{~V}$; potential increment, $0.004 \mathrm{~V}$. 

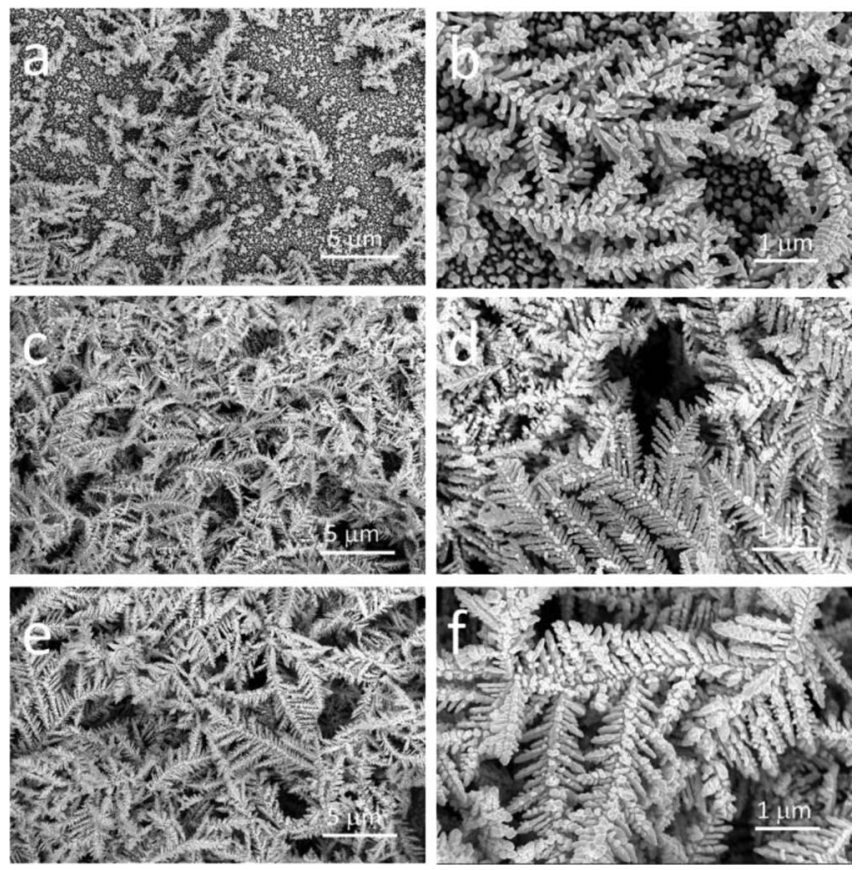

Figure 6. SEM images of gold dendrites electrodeposited on GC electrodes at $-0.8 \mathrm{~V}$ for various time: $1000 \mathrm{~s}(\mathrm{a}, \mathrm{b}), 2000 \mathrm{~s}(\mathrm{c}, \mathrm{d}), 3000 \mathrm{~s}(\mathrm{e}, \mathrm{f})$.

(100) and $\mathrm{Au}(110)$ domains. In this way, $<111>$ is the predominant growth direction during electrodeposition at all time periods.

Figure 6 displays SEM images of Au dendrites electrodeposited for 3 different times. On each surface, very similar hierarchical dendritic branching structures were observed. Figure S10 shows the enhanced gold electrochemical surface area with deposition time. Figure 7 shows the electroanalytical activity of the three gold dendrite surfaces for As (III) analysis. In contrast to the gold spikes electrodes, all three gold dendrites electrodes show comparable sensitivity after normalization to the ECSA, as shown in Figure 7d, indicating that the longer deposition time does not affect As (III) analysis. Lead UPD curves are shown in Figure S11 confirm the polycrystalline feature of all gold dendrites, and give intensities and stripping potentials without significant shifts in the general $\mathrm{Au}$ (111) region after normalizing to the ECSA, confirming a similar surface crystallography at the different deposition times. This explains the comparable sensitivity of the three gold dendrite surfaces, with hierarchical dendritic branching allowing the organic additive to maintain its shape directing behavior, and maintain sensitivity for Arsenic analysis.

\section{Conclusions}

Through a careful study of As (III) analysis on three Au spike morphologies with variable morphology, Au dendrites, and three AuNP shapes that represent the 3 low index planes of $\mathrm{Au}$, we have validated that the $\mathrm{Au}$ (111) facet, or bulk surfaces with effective growth directed deposition gives the best sensitivity for analysis of As (III) by SWASV, which is the most commonly used electroanalytical technique for As (III) analysis. The main factor is the superior slope of the calibration curve for the $\mathrm{Au}$ (111) facet, which displays a cleaner stripping peak for the multi-electron oxidation of As (0) to As (III). The trend in sensitivity is clear, and $\mathrm{Au}$ (111)-rich surfaces also have better limit of detection (LOD) based on the background current, which is another indication of better performance of the $\mathrm{Au}$ (111) facet in the potential range studied in acidic $\mathrm{HNO}_{3}$ solution. It should be noted that in this paper we were most interested in the relative performance of the surfaces under study, and constant conditions were more important than maximising the sensitivity, which could have been done with longer deposition times at low As (III) concentrations to more accurately determine the practical LOD. There are many reviews that compare the sensitivity of surfaces for electrochemical determination of As(III), ${ }^{12,57}$ and although direct comparisons of sensitivities are difficult due to different conditions used papers, the work presented here sheds further light on the reasons for differences amongst Au based surfaces. The precise reason for the improved sensitivity is difficult to determine, and may require further mechanistic studies, however there are some clues from previous studies. The behavior of the (111) facet has been considered theoretically for catalytic processes, and observed in some previous studies on bare nanoparticles. Among the low index planes, the relative surface energies for a face-centered cubic lattice increase in the order $\gamma 111<\gamma 100<\gamma 110$ due to variations in coordination and the density of surface atoms. ${ }^{56}$ Studies on the catalytic activity of Au nanomaterials with controlled morphology are a useful comparison, and catalytic studies of 4-nitroaniline reduction on the same suite of nanoparticles we have used here showed that the
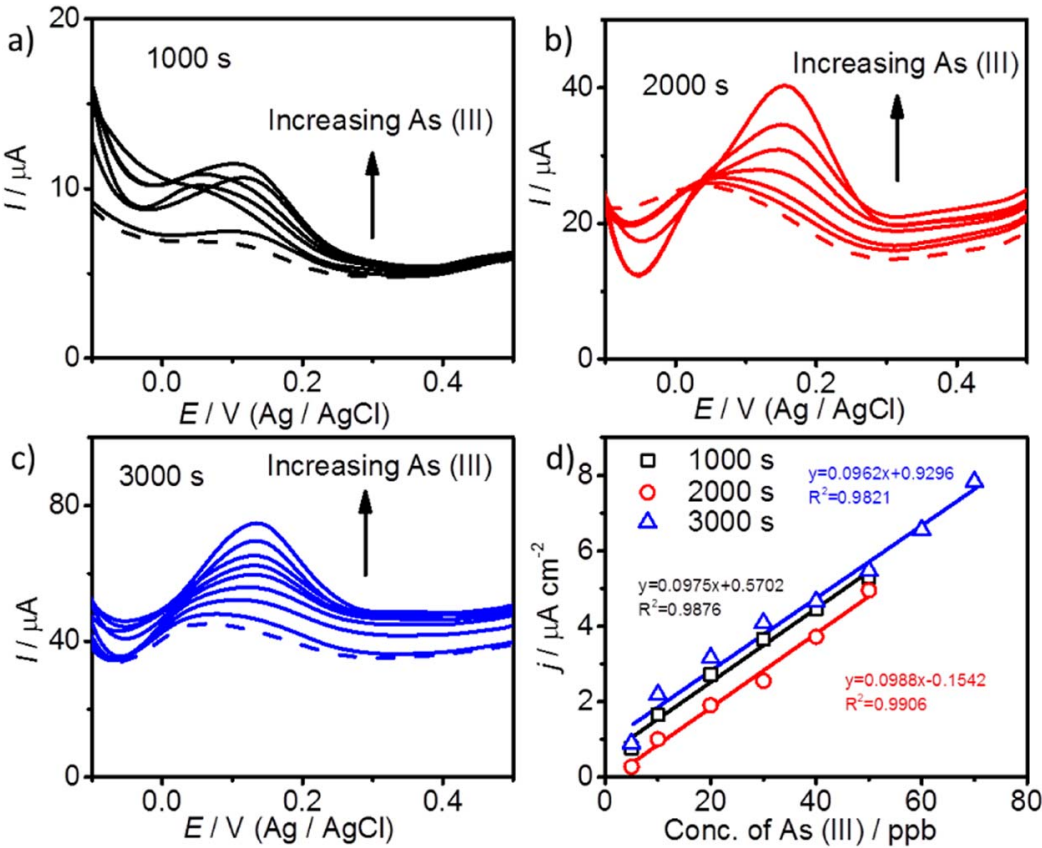

Figure 7. SWASV responses of the gold dendrites electrodes deposited for: a) $1000 \mathrm{~s}$, b) $2000 \mathrm{~s}$, c) $3000 \mathrm{~s}$ for As (III) analysis in $0.5 \mathrm{M} \mathrm{HNO}_{3}$. The dash line refers to the baseline. d) Calibration curves of current density vs. concentrations of As (III) for the three Au dendrites electrodes. Deposition potential, $-0.35 \mathrm{~V}$; deposition time, $100 \mathrm{~s}$; initial potential, $-0.35 \mathrm{~V}$; final potential, $0.7 \mathrm{~V}$; frequency, $15 \mathrm{~Hz}$; amplitude, $0.025 \mathrm{~V}$; potential increment, $0.004 \mathrm{~V}$. 
catalytic activity for the reduction follows the order $(110)>(100)>$ (111), ${ }^{57}$ which has been compared to As (III) analysis on AuNPs. ${ }^{18}$

What we have confirmed in this paper is that $\mathrm{Au}$ (111) rich surfaces, either with Nafion stabilized coated nanoparticles, or controlled spike or dendritic morphologies with larger (111) terraces, give the best sensitivity for electrochemical As (III) detection. The nanoparticle modified electrodes gave the best sensitivity overall, but may not be as practical as the directly electrodeposited surfaces. Further development of sensors for As (III) that are based upon Au should thus be fabricated in a way that maximizes the proportion of (111) facets on the surface, and when deposition of Au structures is used to prepare an electrode, the important parameter of deposition time must be controlled and adjusted, taking into account the additive used. Analysis at relatively low As (III) concentrations, taking into account that the World Health Organization (WHO) specifies $10 \mathrm{ppb}$ as the maximum amount of As (III) in drinking water, will be more stable and precise on $\mathrm{Au}$ (111) surfaces, or surfaces deposited with effective growth directing agents, which will perform better than general polycrystalline Au materials.

\section{Acknowledgments}

We greatly thank RMIT University's RMIT Microscopy and Microanalysis Facility (RMMF) for access to instrument and Australian Research Council for financial support. We acknowledge the staff, especially Dr Matthew Field, Phil Francis, and Peter Rummel in RMMF for their immense scientific and technical assistance. We appreciate Victoria Coyle, Dr Rahul Ram and Dr Ylias Sabri for the help in materials synthesis. B. R. thanks RMIT University for a postgraduate scholarship.

\section{References}

1. B. K. Mandal and K. T. Suzuki, Talanta, 58, 201 (2002).

2. X. Dai, O. Nekrassova, M. E. Hyde, and R. G. Compton, Analytical chemistry, 76, 5924 (2004).

3. A. Salimi, H. Manikhezri, R. Hallaj, and S. Soltanian, Sensor Actuat B-Chem, 129, 246 (2008).

4. D. E. Mays and A. Hussam, Anal Chim Acta, 646, 6 (2009).

5. N. Moghimi, M. Mohapatra, and K. T. Leung, Analytical chemistry, (2015).

6. J. Vandenhecke, M. Waeles, R. D. Riso, and P. Le Corre, Analytical and Bioanalytical Chemistry, 388, 929 (2007).

7. R. Touilloux, M. L. Tercier-Waeber, and E. Bakker, Analyst, 140, 3526 (2015).

8. E. Majid, S. Hrapovic, Y. Liu, K. B. Male, and J. H. Luong, Analytical chemistry, 78, $762(2006)$.

9. A. Cavicchioli, M. A. La-Scalea, and I. G. Gutz, Electroanalysis, 16, 697 (2004).

10. X. A. Dai and R. G. Compton, Analytical Sciences, 22, 567 (2006).

11. B. J. Sanghavi, N. S. Gadhari, P. K. Kalambate, S. P. Karna, and A. K. Srivastava, Microchim Acta, 182, 1473 (2015).

12. J. H. T. Luong, E. Lam, and K. B. Male, Anal Methods-Uk, 6, 6157 (2014).

13. M. Avramov-Ivić, V. Jovanović, G. Vlajnić, and J. Popić, Journal of Electroanalytical Chemistry, 423, 119 (1997).

14. R. R. Adić, N. M. Marković, and V. B. Vešović, Journal of Electroanalytical Chemistry and Interfacial Electrochemistry, 165, 105 (1984).

15. T. J. Schmidt, V. Stamenkovic, M. Arenz, N. M. Markovic, and P. N. Ross Jr, Electrochimica Acta, 47, 3765 (2002).

16. J. Hernandez, J. Solla-Gullon, E. Herrero, A. Aldaz, and J. M. Feliu, J Phys Chem $C, 111,14078$ (2007).

17. M. R. Rahman, T. Okajima, and T. Ohsaka, Analytical chemistry, 82, 9169 (2010).

18. D. -D. Han, S. -S. Li, Z. Guo, X. Chen, J. -H. Liu, and X. -J. Huang, RSC Advances, 6, 30337 (2016).
19. X. Zhang, F. Shi, X. Yu, H. Liu, Y. Fu, Z. Wang, L. Jiang, and X. Li, Journal of the American Chemical Society, 126, 3064 (2004).

20. L. Wang, S. Guo, X. Hu, and S. Dong, Electrochemistry Communications, 10, 95 (2008).

21. H. -X. Ren, X. -J. Huang, O. Yarimaga, Y. -K. Choi, and N. Gu, Journal of colloid and interface science, 334, 103 (2009).

22. M. Li, G. Zhao, R. Geng, and H. Hu, Bioelectrochemistry, 74, 217 (2008).

23. J. -J. Feng, Z. -Y. Lv, S. -F. Qin, A. -Q. Li, Y. Fei, and A. -J. Wang, Electrochimica Acta, 102, 312 (2013).

24. X. Lu, M. S. Yavuz, H. -Y. Tuan, B. A. Korgel, and Y. Xia, Journal of the American Chemical Society, 130, 8900 (2008).

25. N. Zhao, Y. Wei, N. Sun, Q. Chen, J. Bai, L. Zhou, Y. Qin, M. Li, and L. Qi, Langmuir, 24, 991 (2008).

26. B. Seo, S. Choi, and J. Kim, Acs Appl Mater Inter, 3, 441 (2011).

27. D. Seo, J. C. Park, and H. Song, Journal of the American Chemical Society, 128, 14863 (2006).

28. Q. Jiang, Z. Jiang, L. Zhang, H. Lin, N. Yang, H. Li, D. Liu, Z. Xie, and Z. Tian, Nano Research, 4, 612 (2011).

29. Z. -Y. Lv, A. -Q. Li, Y. Fei, Z. Li, J. -R. Chen, A. -J. Wang, and J. -J. Feng, Electrochimica Acta, 109, 136 (2013).

30. T. Huang, F. Meng, and L. Qi, Langmuir, 26, 7582 (2009).

31. J. -J. Feng, A. -Q. Li, Z. Lei, and A. -J. Wang, Acs Appl Mater Inter, 4, 2570 (2012).

32. B. Plowman, S. J. Ippolito, V. Bansal, Y. M. Sabri, A. P. O'Mullane, and S. K. Bhargava, Chem Commun, 5039 (2009).

33. B. J. Plowman, M. Mahajan, A. P. O'Mullane, and S. K. Bhargava, Electrochimica Acta, 55, 8953 (2010).

34. T. H. Lin, C. W. Lin, H. H. Liu, J. T. Sheu, and W. H. Hung, Chem Commun, 47, 2044 (2011)

35. V. E. Coyle, A. E. Kandjani, Y. M. Sabri, and S. K. Bhargava, Electroanalysis, 29, 294 (2017).

36. D. H. Kim, J. W. Heo, M. J. Kim, Y. W. Lee, and S. W. Han, Chem Phys Lett, 468 , 245 (2009).

37. T. K. Sau and C. J. Murphy, Journal of the American Chemical Society, 126, 8648 (2004).

38. H. -L. Wu, C. -H. Kuo, and M. H. Huang, Langmuir, 26, 12307 (2010).

39. A. J. Wain, Electrochimica Acta, 92, 383 (2013).

40. D. Padayachee, V. Golovko, B. Ingham, and A. T. Marshall, Electrochimica Acta, 120, 398 (2014).

41. S. Trasatti and O. Petrii, Pure and applied chemistry, 63, 711 (1991).

42. H. Angerstein-Kozlowska, B. Conway, A. Hamelin, and L. Stoicoviciu, Journal of electroanalytical chemistry and interfacial electrochemistry, 228, 429 (1987).

43. X. Dai and R. G. Compton, Analytical sciences, 22, 567 (2006).

44. G. L. Long and J. D. Winefordner, Analytical Chemistry, 55, 712A (1983).

45. D. J. Anderson, Clinical chemistry, 35, 2152 (1989).

46. A. P. O'Mullane, S. J. Ippolito, Y. M. Sabri, V. Bansal, and S. K. Bhargava, Langmuir, 25, 3845 (2009).

47. M. S. El-Deab, Electrochimica Acta, 54, 3720 (2009).

48. A. Wuttig, M. Yaguchi, K. Motobayashi, M. Osawa, and Y. Surendranath, Proceedings of the National Academy of Sciences, 113, 4585 (2016).

49. C. Rogers, W. S. Perkins, G. Veber, T. E. Williams, R. R. Cloke, and F. R. Fischer, J. Am. Chem. Soc, 139, 4052 (2017).

50. S. Hebié, K. B. Kokoh, K. Servat, and T. W. Napporn, Gold Bulletin, 46 (2013).

51. J. Hernández, J. Solla-Gullón, and E. Herrero, Journal of Electroanalytical Chemistry, 574, 185 (2004)

52. J. Hernández, J. Solla-Gullón, E. Herrero, J. Feliu, and A. Aldaz, Journal of nanoscience and nanotechnology, 9, 2256 (2009).

53. A. J. Motheo, E. R. Gonzalez, G. Tremilliosi-Filho, A. Rakotondrainibe, J. -M. Léger, B. Beden, and C. Lamy, Journal of the Brazilian Chemical Society, 9, 31 (1998).

54. A. Hamelin and J. Lipkowski, Journal of electroanalytical chemistry and interfacial electrochemistry, 171, 317 (1984).

55. A. Hamelin, Journal of electroanalytical chemistry and interfacial electrochemistry, 165, 167 (1984).

56. D. Seo, C. I. Yoo, J. C. Park, S. M. Park, S. Ryu, and H. Song, Angew Chem Int Edit, 47, 763 (2008).

57. S. Kempahanumakkagari, A. Deep, K. Kim, S. Kailasa, and H. Yoon, Biosensors and Bioelectronics, 95, 106 (2017).

58. P. -J. C. Chun-Ya Chiu, Ka-Un Lao, Ching-Wen Liao, and Michael H. Huang, $J$. Phys. Chem. C., 116, 23757 (2012). 